\title{
APROXIMACIÓN AL CONTROL DE LA FASE DE VUELO DE UN DIRIGIBLE
}

\author{
APPROACH TO AIRSHIP CONTROL IN CRUISE PHASE FLIGHT
}

\author{
Leonardo, Solaque Guzmán
}

Ing. Electrónico, PhD., Profesor asistente, Facultad de Ingeniería, Investigador grupo GAV. Universidad Militar Nueva Granada, Bogotá, Colombia, leonardo.solaque@umng.edu.co

\section{Nelson, Muñoz Ceballos}

Ing. Electrónico, MSc., Profesor asistente, Facultad de Ingeniería, Investigador grupo ICARO. Politécnico Jaime Isaza Cadavid, Medellín, Colombia, ndminoz@elpoli.edu.co

Fecha de recepción: 17septiembre de 2007.

Fecha de aprobación: 16 de junio de 2008.

\section{RESUMEN}

El presente trabajo tiene por objetivo el modelo dinámico de un dirigible (como Karma y UrAn), y la elaboración de un conjunto de leyes de control de velocidad, altitud y dirección cuando el dirigible se desplaza en la fase de vuelo crucero. La primera parte del documento presenta un modelo matemático representativo de las principales dinámicas del dirigible. Luego se muestra una descripción de las principales relaciones de las variables, a partir de un modelado e identificación previos, usados para el planteamiento de modelos reducidos, llamados modelos de control. Una segunda parte está dedicada a la formulación de las estrategias de control, basadas en técnicas PID, GPC y una no Lineal. Estas leyes son planteadas sobre la base del desacople de dinámicas para el vuelo lateral y el vuelo longitudinal. Finalmente se incluyen pruebas de simulación y sobre las estructuras reales.

Palabras claves: Plataformas aéreas, dirigible, UAV, control, PID, GPC, Control no lineal, modelado dinámico, velocidad aerodinámica, fases de vuelo.

\section{ABSTRACT}

The aims of this work are the development of a dynamic model for an airship (like Karma and UrAn airships), and the elaboration of a set of base control laws of speed, altitude and heading in cruise flight. The first part of the document presents a mathematical model of the blimp dynamics. The analytic description of the model has been deduced from a dynamic 
and aerodynamic study. In a first time, the model parameters have been taken from precedent works based on wind tunnel experiments. Next to that, simplified models (called control model) were made with classical techniques. A second part is devoted to the formulation of control strategies (like PID, GPC and Non-lineal), based on the decoupling of the velocity, the longitudinal and lateral dynamics. These different control strategies have been validated by numerous simulation tests. The speed, heading and altitude controllers have been tested in two real airship structures.

Key-Words: Aerial platforms, airship, UAV, control, PID, GPC, Non-linear control, dynamic modelling, aerodynamic speed, flight phases.

\section{INTRODUCCIÓN}

El permanente desarrollo en el amplio espectro de la ciencia y tecnología, tal como sensores, actuadores, dispositivos de cómputo y energía, garantizan una larga permanencia de las plataformas más ligeras que el aire. Existe indudablemente un renaciente interés es este dominio, aplicaciones en el transporte de cargas pesadas ${ }^{1} y$ plataformas de gran envergadura a alta altitud para comunicaciones son habilitadas con estas estructuras. Aplicaciones como vigilancia o fotografía aérea, pueden ser realizadas por dirigibles de pequeño tamaño radio-controlados, del orden de pocas decenas de metros cúbicos, brindando gran estabilidad. Ellos son fáciles de operar, vuelan con relativa seguridad a muy bajas altitudes y sobre áreas de particular interés, consumiendo poco combustible, logrando velocidades de decenas de kilómetros por hora y permitiendo mayores tiempos de autonomía. Dentro de sus principales enemigos se encuentran las condiciones meteorológicas, el viento es un agente fuertemente perturbador a sus comportamientos naturales (ver [9]). Se debe notar que las aplicaciones de los dirigibles no tripulados son consideradas cada vez más en el mundo, entre las aplicaciones militares se encuentra la exploración planetaria, como se observa en numerosas contribuciones en las conferencias de los más ligeros que el aire (AIAA) y las convenciones europeas de los dirigibles [14]. La primera mención de los desarrollos de plataformas no tripuladas y autónomas como los dirigibles, se encuentra en la literatura cerca de las década de los 90's [4], pero es solo recientemente que varios proyectos han mostrado logros eficaces. Uno de los proyectos más avanzados en este dominio es Aurora, un proyecto sostenido por el Instituto de la Tecnología e Información de Campinas, Brasil, dedicado principalmente al control del vuelo [7].

Uno de los principales problemas que se deben enfrentar es el control de los prototipos, ya que cuando se pretende lograr un dirigible con habilidades de autonomía surgen

tal como el proyecto ATG Skycats -revisado a la fecha, información sobre este proyecto es disponible en www.worldskycat.com 
dificultades ocasionadas por las no-linealidades en la dinámica de estos sistemas y por el viento como gran perturbador en el desplazamiento durante un vuelo. El trabajo se centra en la síntesis de los controles tipo PID, GPC y No-lineales para la velocidad, altitud y dirección, cuando el dirigible se desplaza a una altitud constante y en línea recta (lo que equivale a un punto de operación dentro de la fase de vuelo crucero). Para esto, se hace un estudio previo del modelo dinámico del dirigible y las formas de encontrar modelos reducidos o de menor complejidad.

Este documento se encuentra organizado de la siguiente manera: la sección 1 presenta un completo modelo dinámico del dirigible, con simplificaciones para la obtención de modelos que permitan la síntesis de las leyes de control. Sobre la hipótesis de una velocidad regulada a un valor constante (hipótesis de fase de vuelo crucero), se permite el desacople de las dinámicas de vuelo lateral y longitudinal, permitiendo expresar la dinámica global en la fase crucero, como dos sub-modelos. De esta manera el control del dirigible puede hacerse a través de tres reguladores independientes, uno dedicado a la velocidad de desplazamiento, otro sobre la altitud y el último sobre la dirección, los cuales se presentan la sección 2. A lo largo de las secciones se incluyen referencias a trabajos previos, así como resultados experimentales obtenidos con dos plataformas diferentes de dirigibles (ver fig. 1). Finalmente se presentan algunas conclusiones.

\section{MODELADO DEL DIRIGIBLE}

Las estructuras consideradas tienen una estructura clásica en 'forma de cigarra' (Fig. 1). Estos robots cuentan con: los motores de empuje principal, montados sobre un eje vectorizado, de manera que se habilite el despegue en forma vertical y provea sustentación adicional para velocidades pequeñas, cuando la sustentación aerodinámica no es suficiente. Las superficies móviles (alerones) posibilitan el control en los planos longitudinal y lateral, y un rotor trasero transmite un empuje adicional que permite el control lateral del dirigible a velocidades pequeñas y cuando los alerones no son suficientes.
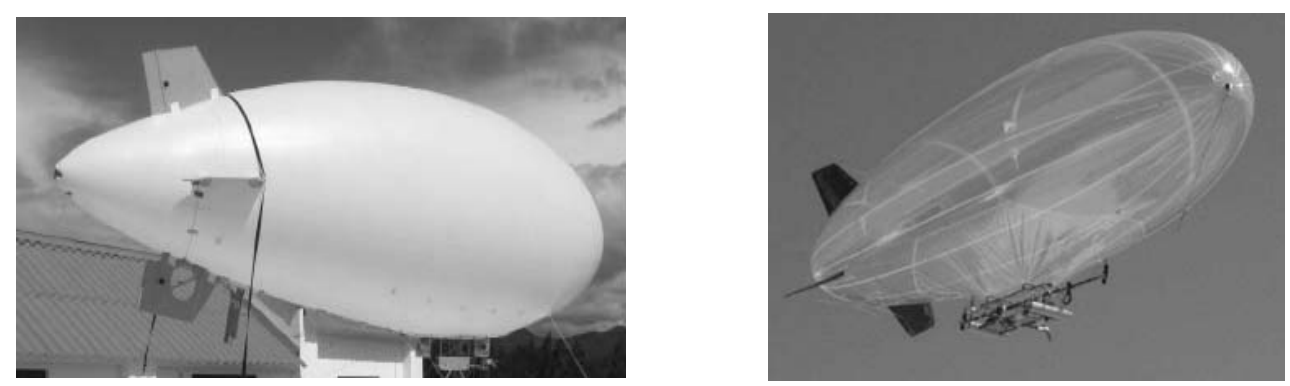

Figura 1. Los dirigibles usados. UrAn (28 m3) y Karma (18 m3), respectivamente. 


\subsection{SISTEMAS DE REFERENCIA}

Para describir el movimiento de un dirigible es necesario tener en cuenta tres referenciales (Fig. 2): el eje global $R_{o}$ fijo a un punto arbitrario sobre la tierra, orientado según la convención NED; el marco fijo al cuerpo del dirigible $R_{d}$, que tiene su origen en el centro volumétrico (CV) de la envoltura [17]; (CV se escoge como el origen de este marco porque coincide con el centro de empuje, donde la fuerza de sustentación aerostática es aplicada) y finalmente, el marco aerodinámico $R_{a}$ que está alineado con la dirección del movimiento del dirigible. El eje $x_{d}$ de $R_{d}$ coincide con el eje de simetría de la envoltura, el plano $\left(x_{d}, z_{d}\right)$ coincide con el plano longitudinal y la orientación de $R_{d}$ con respecto a $R_{o}$ está dada por los ángulos de Euler 'roll' $\phi$, 'pitch' $\theta$ y 'yaw' $\psi$. El eje $x_{a}$ de $R_{a}$ coincide con la velocidad aerodinámica del dirigible $V_{A \mid R_{a}}=\left(M_{d}^{a}\right)^{-1}\left(v_{d l}-v_{w}\right)$, donde $v_{d l}$ y $v_{w}$ respectivamente denotan las velocidades del dirigible y la velocidad del viento con respecto a $R_{o}$. $\alpha$ es el ángulo de ataque y $\beta$ es el ángulo de derrapaje en el plano $\left(x_{d}, y_{d}\right)$. La matriz de rotación $M_{d}^{o}$ entre el marco coordenado global $R_{o}$ y el marco coordenado local $R_{d}$ esta dado por:

$$
M_{o}^{d}=\left(\begin{array}{ccc}
c \psi c \theta & -s \psi c \phi+s \phi c \psi s \theta & s \phi s \psi+s \theta c \psi c \phi \\
c \theta s \psi & c \psi c \phi+s \theta s \psi s \phi & -c \psi s \phi+c \phi s \theta s \psi \\
-s \theta & c \theta s \phi & c \theta c \phi
\end{array}\right)
$$

y la matriz de transformación entre el marco coordenado local $R_{d}$ y el marco coordenado aeronáutico $R_{a}$ es:

$$
M_{d}^{a}=\left(\begin{array}{ccc}
c \alpha c \beta & -c \alpha s \beta & -s \alpha \\
s \beta & c \beta & 0 \\
s \alpha c \beta & -s \alpha s \beta & c \alpha
\end{array}\right)
$$

donde $S x($ resp. $C x)$ denota la función $\sin (x)($ resp. $\cos (x))$.

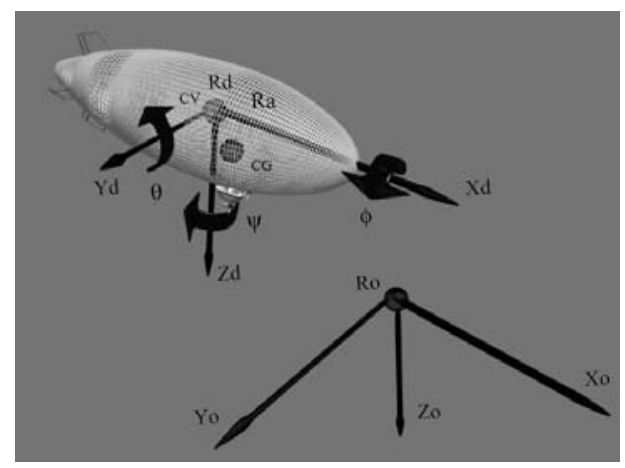

Figura 2. Referenciales del dirigible 


\subsection{MODELO DINÁMICO}

El modelo dinámico del dirigible se establece sobre la base de las siguientes hipótesis:

- La envoltura es considerada como un sólido: los fenómenos areo-elásticos y el movimiento del helio dentro de la envoltura son ignorados;

- La masa del dirigible y su volumen son considerados constantes;

- $\quad$ El dirigible desplaza volumen: los fenómenos inducidos por este desplazamiento producen fenómenos de masas e inercias adicionadas;

- La posición del centro de empuje de la fuerza de sustentación aerostática coincide con el centro de volumen $\mathbf{C V}$;

- La velocidad de un dirigible pequeño es generalmente baja (bajo número de match), los acoplamientos entre las dinámicas y los fenómenos térmicos son despreciables al igual que la modificación de la densidad del aire debida al movimiento del sistema;

- $\quad$ La tierra es considerada plana sobre el área de vuelo.

Las anteriores hipótesis son razonables para los dirigibles considerados, permitiendo aplicar la teoría de la mecánica de cuerpo rígido. A través del uso de las leyes de Newton, las teorías de aerodinámica [3] con las leyes de Kirshoff's y Bryson's, el modelo dinámico con respecto al referencial $R_{d}$, conocido como modelo para simulación, pueden describirse como:

$$
M_{d} \dot{\vec{v}}=-T_{d}\left(v_{d l r}\right)+T_{a}\left(v_{A}\right)+g_{a}+T_{p}
$$

Donde:

- $\quad v$ es el vector de estado cuyas componentes son las velocidades lineales $v_{d}=[u, v, w]^{T}$ y las velocidades angulares $v_{m}=[p, q, r]^{T}$. Para propósitos de la realización de cualquier control, estas variables deben ser expresadas en $R_{o}$, lugar donde se referencia generalmente las medidas del GPS y la central inercial.

- La matriz $M_{d}$ de $6 X 6$ esta compuesta de masas, inercias y términos de acoplamiento:

$$
M_{d}=\left(\begin{array}{cccccc}
m & 0 & 0 & 0 & m z_{g} & 0 \\
0 & m & 0 & -m z_{g} & 0 & m x_{g} \\
0 & 0 & m & 0 & -m x_{g} & 0 \\
0 & -m z_{g} & 0 & I_{x} & 0 & -I_{x z} \\
m z_{g} & 0 & -m x_{g} & 0 & I_{y} & 0 \\
0 & m x_{g} & 0 & -I_{x z} & 0 & I_{z}
\end{array}\right)
$$

Donde $\left(x_{g}, z_{g}\right)$ es la posición del centro de gravedad. El término $y_{g}$ es cero ya que coincide con la misma posición del centro volumétrico (CV).

- $\quad T_{d}$ es el vector de fuerza dinámica y contiene la fuerza centrífuga y de Coriolis: 


$$
T_{d}\left(v_{d}\right)=\left(\begin{array}{c}
m w q-m v r-m x_{g} q^{2}+m z_{g} p r-m x_{g} r^{2} \\
m u r-m w p m z_{g} q r+m x_{g} p q \\
m v p-m u q-m z_{g} p^{2}+m x_{g} p r-m z_{g} q^{2} \\
-m z_{g} u r+m z_{g} w p-I_{x z} p q-\left(I_{y}-I_{z}\right) q r \\
-m z_{g} v r-m x_{g} v p m z_{g} w q+m x_{g} u q+I_{x z}\left(p^{2}-r^{2}\right)-\left(I_{z}-I_{x}\right) p r \\
-m x_{g} w p+m x_{g} u r-\left(I_{x}-I_{y}\right) p q+I_{x z} q r
\end{array}\right)
$$

- $\quad g_{a}$ contiene las fuerzas debidas a la gravedad y a la fuerza de empuje ( $F_{G}$ es el peso del dirigible, $F_{B}$ es la fuerza de empuje de sustentación):

$$
g_{a}=\left(\begin{array}{c}
-\left(F_{G}-F_{B}\right) s \theta \\
\left(F_{G}-F_{B}\right) c \theta s \phi \\
\left(F_{G}-F_{B}\right) c \theta c \phi \\
-z_{g} F_{G} c \theta s \phi \\
-z_{g} F_{G} s \theta-\left(x_{g} F_{G}-x_{c} F_{B}\right) c \theta c \phi \\
\left(x_{g} F_{G}-x_{c} F_{B}\right) c \theta s \phi
\end{array}\right)
$$

Donde $x_{c}$ representa un desplazamiento del punto donde se produce la fuerza de empuje $F_{B}$. Según hipótesis mencionadas $x_{c=} 0$.

- $\quad T_{p}$ representa las fuerzas de control aplicadas al dirigible: contienen los torques de los propulsores vectorizados $F_{M}$ en $\left(O_{x}, O_{z}\right)$ y la fuerza de empuje del rotor de cola $F_{r c}$ en $\left(x_{r c}, z_{r c}\right)$. La dirección de los propulsores en función de $\mu$, es ajustable en el plano longitudinal y la dirección del rotor de cola en el plano lateral.

$$
T_{p}=\left(\begin{array}{c}
F_{M} c \mu \\
F_{r c} \\
-F_{M} s \mu \\
F_{r c} z_{r c} \\
F_{M} O_{z} c \mu+F_{M} O_{x} s \mu \\
F_{r c} x_{r c}
\end{array}\right)
$$

- $\quad T_{a=} A v_{A}{ }^{\prime}-D\left(v_{d r}\right) v_{A}+T_{s t a}\left(v_{a}^{2}\right)$ es el vector aerodinámico, donde:

o A es la matriz de masas e inercias adicionadas en el centro de gravedad (CG), y los términos de acoplamiento (masas e inercias virtuales - [9], [12] presenta una manera de estimar estos coeficientes): 


$$
A v_{A}=\left(\begin{array}{cccccc}
a_{11} & 0 & 0 & 0 & a_{15} & 0 \\
0 & a_{22} & 0 & a_{24} & 0 & a_{26} \\
0 & 0 & a_{33} & 0 & a_{35} & 0 \\
0 & a_{42} & 0 & a_{44} & 0 & a_{46} \\
a_{51} & 0 & a_{53} & 0 & a_{55} & 0 \\
0 & a_{62} & 0 & a_{64} & 0 & a_{66}
\end{array}\right)\left(\begin{array}{c}
\dot{V}_{X_{a}} \\
\dot{V}_{Y_{a}} \\
\dot{V}_{Z_{a}} \\
\dot{p} \\
\dot{q} \\
\dot{r}
\end{array}\right)
$$

o $v_{A}=\left[v_{a}, v_{m}\right]$, donde $\overrightarrow{v_{a}}=\overrightarrow{v_{d}}-\overrightarrow{v_{w}}$ es el vector de velocidad traslacional, $\overrightarrow{v_{w}}=\left[u_{w}, v_{w}, w_{w}\right]^{T}$ es la velocidad del viento en $R_{d}$. En Azinheira [2] se muestra un estudio de la influencia del viento sobre el dirigible.

o $D\left(v_{d r}\right) v_{A}$ es el vector que contiene los términos de la fuerza centrífuga y de Corilis:

$$
D_{1}\left(v_{d} r\right) v_{A}=\left(\begin{array}{cccccc}
0 & a_{22} r & -a_{33} q & a_{24} r & -a_{35} q & a_{26} r \\
D_{1 z 1} & 0 & a_{33} p & a_{35} q & -a_{15} q & 0 \\
\left(a_{11}-x m_{22}\right) q & -a_{22} p & 0 & -a_{24} p-a_{26} r & a_{15} q & 0 \\
D_{1 z 2} & -\left(a_{62}+a_{35}\right) q & D_{1 z 3} & -a_{64} q & \left(a_{55}-a_{66}\right) r & 0 \\
\left(a_{35}+x^{2} m_{22}\right) q & -a_{42} r a_{62} p & -a_{15} q & D_{1 z 5} & 0 & -a_{64} r \\
D_{1 z 4} & \left(a_{15}+a_{42}\right) q & -a_{53} p & \left(a_{44}-a_{55}\right) q & a_{46} r & 0
\end{array}\right)
$$

Donde:

$D_{1 z 1}=p m_{13}+r\left(x m_{11}-a_{11}\right), D_{1 z 2}=p m_{33}+r\left(a 15+x m_{13}\right), D_{1 z 3}=\left(a_{62}+a_{35}\right) r+a_{24} P, D_{174}=\left(a_{51}+a_{24} x m_{13}\right)$ $p-\left(a_{26}-x^{2} m_{11}\right) r$, y $D_{175}=a_{64} p+\left(a_{66}-a_{44}\right) r$. Bonnet [3] presenta como se determinan estos coeficientes.

- $\quad T_{\text {sat }}\left(v_{a}^{2}\right)$ representa las fuerzas aerodinámicas y momentos en CG, proporcionales a la forma de la envoltura y al cuadrado de la velocidad aerodinámica.

$$
T_{s t a}\left(v_{a}^{2}\right)=\left(\begin{array}{c}
\frac{1}{2} \rho v_{a}^{2} S_{r e f} C_{T} \\
\frac{1}{2} \rho v_{a}^{2} S_{r e f} C_{L} \\
\frac{1}{2} \rho v_{a}^{2} S_{r e f} C_{N} \\
-\frac{1}{2} \rho v_{a}^{2} S_{r e f} L_{r e f} C_{l N} \\
-\frac{1}{2} \rho v_{a}^{2} S_{r e f} L_{r e f} C_{m N} \\
-\frac{1}{2} \rho v_{a}^{2} S_{r e f} L_{r e f} C_{n N}
\end{array}\right)=\left(\begin{array}{c}
F_{x} \\
F_{y} \\
F_{z} \\
L_{0} \\
M_{0} \\
N_{0}
\end{array}\right) \text { (10) }
$$

Donde: $S_{r e f}$ y $L_{r e f}$ dependen de la geometría del dirigible. $C_{T}, C_{L}, C_{N}, C_{I N}, C_{m N}$ y $C_{m M}$ son respectivamente, los coeficientes estacionarios tangencial, normal, lateral, roll, pitch y yaw. 


\subsection{MODELOS SIMPLIFICADOS}

Con el fin de obtener un modelo dinámico suficientemente representativo de las principales dinámicas del dirigible y de menor complejidad para la síntesis de las leyes de control (modelos de control), es necesario hacer simplificaciones adicionales de forma que el domino de operación se restrinja logrando desacoplar el vuelo lateral y longitudinal. En el caso de un vuelo crucero en ausencia del viento, la ecuación que representa la dinámica de la velocidad en función de la fuerza de los propulsores es:

$$
\dot{u}=\frac{1}{\left(m-a_{11}\right)}\left(F_{m} \cos \mu+\frac{1}{2} \rho V_{a}^{2} \operatorname{SrefC}_{T}\right)
$$

Provisto de una velocidad constante $\left(u=u_{o}\right)$ y suponiendo que se desplaza en línea recta $\left(\delta_{\mathrm{g}}=0, v=0\right)$, el modelo que muestra la evolución de la altitud puede ser simplificado. Las variables involucradas en esta dinámica son $z, w, u, \theta, q$ y $\delta_{e}$ y: considerando que $w<<u$ y, $\alpha \approx 0$ se tiene.

$$
\begin{gathered}
\dot{z}=-u \sin \theta \\
\dot{\theta}=q \\
\dot{q}=\frac{N U M}{I_{y}-a_{55}}
\end{gathered}
$$

Donde:

$$
\begin{gathered}
N U M=\left(\left(m x_{g}-a_{35}-x^{2} m_{22}\right) u q-z_{g} F_{G} \sin \theta-\left(x_{g} F_{G}-x_{c} F_{B}\right) \cos \theta+F_{m} O_{z} \cos \mu+\ldots\right. \\
\left.\ldots+F_{m} O_{x} \sin \mu-\frac{1}{2} \rho u^{2} S_{r e f} L_{r e f} C_{m N}\right)
\end{gathered}
$$

Linealizando alrededor del punto de operación (condiciones de vuelo crucero, $u=c t e, \theta$ pequeño), $-\left(x_{g} F_{G}-x_{C} F_{B}\right) \cos \theta+F_{m} O_{z} \cos \mu \approx 0 \rightarrow \sin \theta$ y $\cos \theta \approx 1$, se tiene:

$$
\begin{gathered}
\dot{z}=-u \sin \theta \\
\dot{\theta}=q \\
\dot{q}=k_{2 \mid \delta_{e}} q+k_{1 \mid \delta_{e}} \theta+k_{3 \mid \delta_{e}} \delta_{e}
\end{gathered}
$$

Donde:

$$
k_{2 \mid \delta_{e}}=\frac{\left(m x_{g}-a_{35} x^{2} m_{22}\right) u}{I_{y}-a_{55}}, \quad k_{1 \mid \delta_{e}} \approx \frac{-z_{g} F_{G}}{I_{y}-a_{55}}, \text { y } k_{3 \mid \delta_{e}} \approx \frac{\rho u^{2} S_{r e f} L_{r e f} C_{m N}}{2\left(I_{y}-a_{55}\right)}
$$


La ecuación (13) corresponde a un sistema de control de segundo orden para $\theta$ y tercer orden para $z$. Considerando la dinámica de $\theta$ similar a un primer orden, el modelo de altitud puede ser visto como un primer orden más un integrador puro.

Ahora al considerar la velocidad constante en un plano $(x, y)$ con $\delta_{e}=0$ y w $=0$, las ecuaciones dinámicas del dirigible y su posición en el plano horizontal se pueden escribir como:

$$
\begin{gathered}
\dot{x}=u \cos \psi-v \sin \psi \\
\dot{y}=u \sin \psi-v \cos \psi \\
\dot{\psi}=r \\
\dot{r}=\frac{u r\left(m x_{g}+a_{26}-x^{2} m_{11}\right)+F_{r c} x_{r c}-\frac{1}{2} \rho u^{2} S_{r e f} L_{r e f} C_{n N}}{\left(I_{z}-a_{66}\right)}
\end{gathered}
$$

Las variables involucradas en este modelo son $x, y, u, v, y, r$ y $\delta_{g}$. Considerando $v<<u, \beta \approx 0$ y $F_{r c}=0$, las ecuaciones (14) se simplifican a:

$$
\begin{gathered}
\dot{x}=u \cos \psi \\
\dot{y}=u \sin \psi \\
\dot{\psi}=r \\
\dot{r}=k_{2 \mid \delta_{g}} r+k_{1 \mid \delta_{g}} \delta_{g}
\end{gathered}
$$

Note que los coeficientes $k_{1 \mid \delta_{g}}$ y $k_{2 \mid \delta_{g}}$ pueden ser determinados de manera similar al modelo reducido de altitud (en Fossard et al [11] se encuentra una aplicación similar de la dinámica simplificada para un modelo de aeroplanos en fase de vuelo crucero).

\section{CONTROL}

Con modelos reducidos, suficientemente representativos de la dinámica del dirigible, se realizó una estimación de los coeficientes del sistema y se atacó el problema de control para el desplazamiento a velocidad constante. En general, se encuentran en la literatura, contribuciones al control de dirigibles, básicamente con resultados en simulación. Elfes et al [10] presentan una arquitectura de control donde el 'yaw', 'pitch' y la altitud son controlados sobre la base de controles PI y PID adaptivos. Paiva et al [7] describe una estrategia de control con un PID para la velocidad en el plano longitudinal y un PD para la altitud y la dirección. Azinheira et al [1] un regulador para la dirección basado en técnicas de control robusto tipo $H_{\infty}$, para el seguimiento de trayectorias. Otros autores optan por control difuso, tal como Zhenbang et al [18], quien describe un control de dirección para la navegación, basado en control difuso optimizado por algoritmos genéticos. Díaz et al [8] proponen un compensador distribuido paralelo para la fase de vuelo crucero, basado en 
técnicas difusas. Hygounenc y Souères [12] proponen un control con estrategias de backstepping.

En esta sección se presenta el análisis de la ley de control basado en técnicas PID y considerando los sistemas como SISO (Single Input-Single Output). Estos controles se plantean sobre el principio de tener desacoplado el vuelo longitudinal y lateral (ver [12]). Los controles se basan en: 1 ) un control para la velocidad aerodinámica es el resultado de los propulsores principales; 2) las superficies móviles en configuración de-simétrica $\delta_{e}$ controlan la altitud, y 3 ) el control de dirección es resultado de la acción de alerones en configuración simétrica $\delta_{g}$.

Planteadas las hipótesis anteriores, el diseño de las leyes de control, debe ser tal que el dirigible en sus estados principales siga tan cerrado como pueda a las señales de referencias fijadas $u_{r}, z_{r}$ y $\psi_{r}$, respetando las hipótesis de vuelo crucero y manteniendo la validez de los modelos simplificados o en otras palabras, respetando el comportamiento del sistema en lazo cerrado.

\subsection{CONTROL PID}

Cada modelo simplificado mostrado en la sección 1.1.3. se puede representar por:

$$
\begin{gathered}
\dot{x}=A x+B u \\
y=C x
\end{gathered}
$$

donde $x$ es un $n X 1$ vector de estado, $y$ es un $p X 1$ vector de salida y $u$ es un $q X 1$ vector de entrada ( $A, B, C$ y $D$ son respectivamente, $n X n, n X P, q X n$, y $q X p$, matrices). La ley de control $u$ definida por un control PID puede ser descrita como:

$$
u=K_{p} u_{m}+K_{i} \int\left(u_{r e f}-u_{m}\right) d t+K_{d} \frac{d u_{m}}{d t}
$$

Donde $u_{\text {ref }}$ es la referencia y $u_{m}$ es la variable de control. Las ganancias proporcional, integral y derivativo $k p$, $k i$ y $k d$ pueden ser determinadas gracias a la realimentación de estado y la teoría de asignación de polos. Finalmente, es recomendado usar una estrategia anti-windup para habilitar la saturación al término integral.

\subsubsection{Control de Velocidad}

Con el modelo de lazo abierto para la velocidad aerodinámica escrito como: 


$$
H(s)=\frac{v_{a}(s)}{T(s)}=\frac{\kappa}{\tau s+1}
$$

y la señal de control para los propulsores $(T)$ dada por:

$$
T(s)=k_{p} v_{a}+\frac{k_{i}}{s}\left(v_{r}-v_{a}\right)
$$

Donde $v_{r}$ es la señal de referencia para la velocidad, el polinomio característico en lazo cerrado queda descrito por:

$$
s^{2}-\frac{1}{\tau}\left(1+\kappa k_{p}\right) s+\frac{\kappa}{\tau} k_{i}
$$

Ahora, definiendo un polinomio $s^{2}+2 \zeta \varpi_{n} s+{\varpi_{n}}^{2}$ basado en los criterios de desempeño e igualando a la eq. (20), los coeficientes del PI $\left(k_{p}\right.$ y $\left.k_{i}\right)$ pueden ser determinados.

\subsubsection{Controles de altitud y dirección}

Con un modelo de control para la altitud escrito como:

$$
H(s)=\frac{z(s)}{\delta_{e}(s)}=\frac{k_{z} k_{3}}{s^{3}-k_{2} s^{2}-k_{1} s}
$$

y la señal de control dada por un PID:

$$
\delta_{e}(s)=k_{p} z+\frac{k_{i}}{s}\left(z_{r}-z\right)+k_{d} \theta+k_{2 d} q
$$

Donde $z_{r}$ es la referencia de la altitud, el polinomio característico en lazo cerrado es:

$$
s^{4}-s^{3}\left(k_{2}+k_{3} k_{2 d}\right)-s^{2}\left(k_{1}+k_{3} k_{d}\right)-s\left(k_{p} k_{3} k_{z}\right)+k_{z} k_{3} k_{i}
$$

Basado en los criterios de desempeño para este regulador, el polinomio característico queda:

$$
\left(s^{2}+2 \xi \varpi_{n} s+\varpi_{n}^{2}\right)\left(s+1 / \tau_{1}\right)\left(s+1 / \tau_{2}\right)
$$


Igualando este con la eq. (23), se pueden determinar los coeficientes $k_{p}, k_{i}, k_{d}, y k_{2 d}$ para el control de altitud. Las ganancias del control de dirección se pueden obtener con estos procedimientos.

\subsubsection{Resultados en simulación}

La figura 3 muestra los resultados en simulación de los tres controles PID's, aplicados sobre el modelo de simulación (recordar que este modelo contiene la mayor descripción de la dinámica del dirigible). La estabilización de cada estado de salida del sistema se presenta manifestando un poco de acoplamiento entre las acciones de los reguladores. Existe una pérdida de altitud cuando el dirigible toma las curvas, ya que por seguridad éste se deja ligeramente más pesado (fuerza de gravedad es ligeramente mayor que la fuerza de empuje).
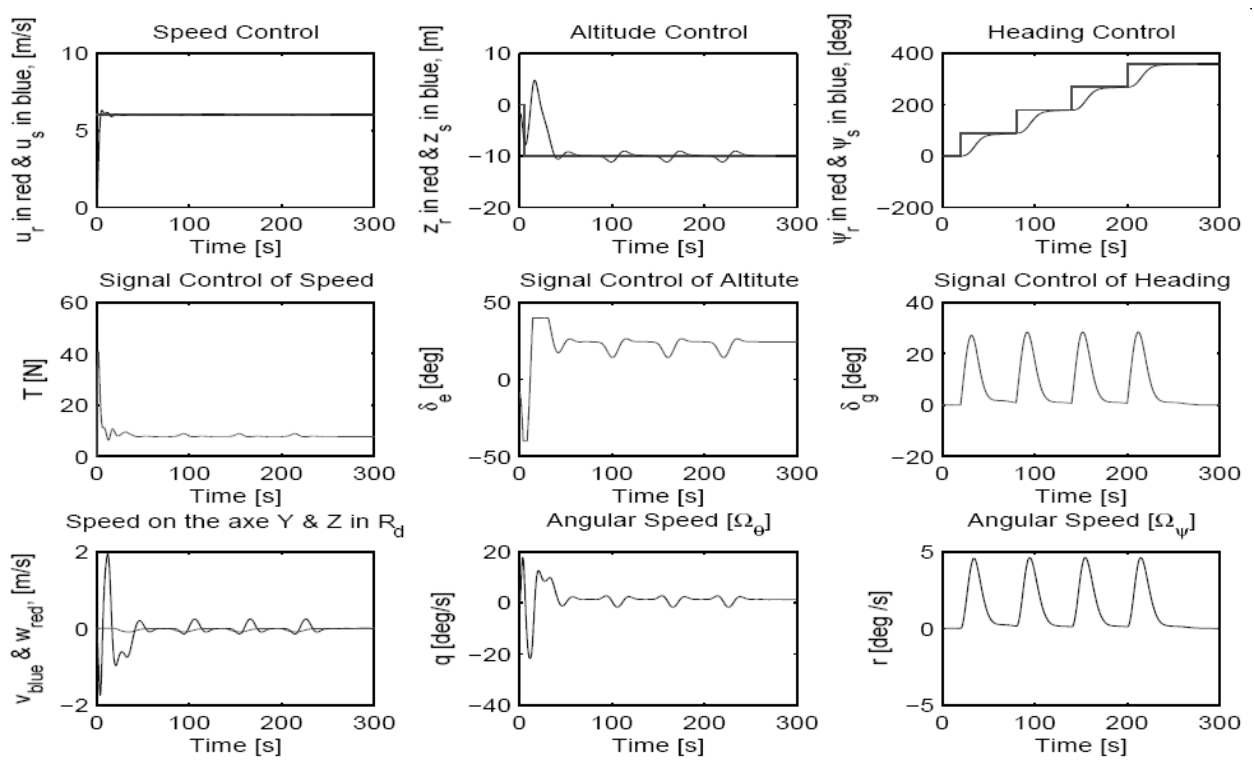

Figura 3. Resultados de los controles PID's: de arriba a abajo, se presenta la evolución de los sistemas, los controles y las derivadas de las variables de control

\subsection{CONTROL PREDICTIVO GENERALIZADO - GPC}

El control predictivo generalizado fue introducido por Clarke et al [6], y se popularizó en la industria y la academia [5]. Es una clase de control predictivo basado sobre la minimización de una función objetivo para la generación de la ley de control. El sistema SISO puede ser descrito de la siguiente forma:

$$
A\left(z^{-1}\right) y(t)=z_{-d} B\left(z^{-1}\right) u(t-1)+C\left(z^{-1}\right) e(t)
$$


donde $u(t)$ y $y(t)$ son las señales de control y salida del sistema, y e $(t)$ representa el ruido blanco con media cero. $A, B$ y $C$ son polinomios en $z$ y $d$ es el tiempo muerto del sistema. Este modelo es llamado modelo 'Controller Auto-Regressive Moving-Average (CARMA)'. Cuando las perturbaciones son no-estacionarios, el modelo CARMA no es apropiado y se debe considerar el modelo integral de CARMA (CARIMA) [6]. Considerando notación similar a la de Camacho [5], el modelo CARIMA se convierte en:

$$
A\left(z^{-1}\right) y(t)=z_{-d} B\left(z^{-1}\right) u(t-1)+C\left(z^{-1}\right) \frac{e(t)}{\Delta}
$$

con $. \Delta=1-z^{-1}$

El algoritmo GPC consiste en aplicar una secuencia de control que minimiza la función de costo siguiente:

$$
\begin{gathered}
J\left(N_{1}, N_{2}, N_{u}\right)=E\left\{\sum_{j=N_{1}}^{N_{2}} \delta(j)[y(t+j \mid t)-w(t+j)]^{2}\right. \\
\left.+\sum_{j=1}^{N_{u}} \lambda(j)[\Delta u(t+j-1)]^{2}\right\}
\end{gathered}
$$

Donde $E\left\{{ }^{\star}\right\}$ es la esperanza y $y(t+j \mid t)$ es la j-esima predicción de la salida del sistema, sobre un tiempo futuro, $N_{1}$ y $N_{2}$ son el mínimo y máximo de la función de costo sobre un horizonte, $N_{u}$ es el horizonte de control, $\delta(j)$ y $\lambda(j)$ son los pesos de la secuencia y $w(t+j)$ es la trayectoria de referencia futura.

El objetivo del control GPC es encontrar una secuencia de control futura $u(t), u(t+1), \ldots$, tal que la salida futura del sistema $y(t+j)$ pueda seguir tan cerca como sea posible a $w(t+j)$, considerando siempre la minimización de la función de costo $J\left(N_{1}, N_{2}, N_{3}\right)$.

\subsubsection{Control de velocidad}

El equivalente discreto del modelo de velocidad simplificado, con un tiempo de muestreo $t_{\mathrm{s}}=0.1 \mathrm{~s}$, viene representado por:

$$
H\left(z^{-1}\right)=\frac{0.0198 z^{-1}}{1-0.9802 z^{-1}}
$$

Note que para este sistema, el tiempo muerto $d$ es igual a cero y el polinomio asociado al ruido es 1 .

Ahora con el algoritmo presentado por Camacho [5] y los valores de los parámetros $N_{1}=1$, $N_{2}=N=10$ y $\lambda=1.2$, la ley de control viene dada por: 


$$
\begin{gathered}
T(k)=T(k-1)-4.7404 v_{a}(k)+4.0845 v_{a}(k-1) \\
+0.0151 v_{a \mid r e f}(k+1)+0.0288 v_{a \mid r e f}(k+2) \\
+\cdots+0.1084 v_{a \mid r e f}(k+10)
\end{gathered}
$$

\subsubsection{Control de altitud}

Consideremos el siguiente modelo discreto para la dinámica de la altitud:

$$
H\left(z^{-1}\right)=\frac{0.000603+0.002424 z^{-1}+0.000609 z^{-2}}{1-3.021 z^{-1}+3.041 z^{-2}-1.02 z^{-3}}
$$

Si $d=0, C\left(z^{-1}\right)=1$ y los valores de los parámetros $N_{1}=1, N_{2}=N=10$ y $\lambda=15$, el control calculado viene dado por:

$$
\begin{gathered}
\delta_{e}(k)=0.9484 \delta_{e}(k-1)-0.0516 \delta_{e}(k-2)-24.2363 z(k) \\
+65.6447 z(k-1)-59.8995 z(k-2)+18.3751 z(k-3) \\
+0.00004 z_{r e f}(k+1)+0.00031 z_{r e f}(k+2) \\
+\cdots+0.0384 z_{r e f}(k+10)
\end{gathered}
$$

\subsubsection{Control de dirección}

Considerando el modelo discreto para la dinámica del la dirección del dirigible:

$$
H\left(z^{-1}\right)=\frac{0.001839 z^{-1}+0.001722 z^{-2} \underline{z}}{1-1.822 z^{-1}+0.8219 z^{-2}}
$$

y conociendo $d=0, C(z-1)=1$, los valores de los parámetros $N_{1}=1, N_{2}=N=10$ y $\lambda=0.6$, la ley de control para la dirección del dirigible se puede escribir como:

$$
\begin{gathered}
\delta_{g}(k)=0.9717 \delta_{g}(k-1)-0.0282 \delta_{g}(k-2)-38.5727 \psi(k) \\
+64.3467 \psi(k-1)-26.4822 \psi(k-2)+0.0029 \psi_{\text {ref }}(k+1) \\
+0.0108 \psi_{\text {ref }}(k+2)+\cdots+0.1609 \psi_{\text {ref }}(k+10)
\end{gathered}
$$

\subsubsection{Resultados de simulación}

La figura 4 presenta los resultados del control GPC, con el mismo modelo e idénticas referencias presentadas en la sección 2.1. 


\subsection{REGULADORES NO LINEALES TIPO PRIMER ORDEN}

Esta técnica contempla los sistemas tipo SISO (Single Input - Single Output) y NLTI (NonLinear Time Invariant) o modelos con variaciones paramétricas lentas, tal como se pueden considerar los modelos simplificados (o comportamiento del sistema en la fase crucero). La presente sección es dedica a las leyes de control generadas por la técnica de control de primer orden (FOC) por realimentación de estado, la cual es una técnica dedicada al estudio de los sistemas no lineales en la vecindad del punto de equilibrio del sistema.
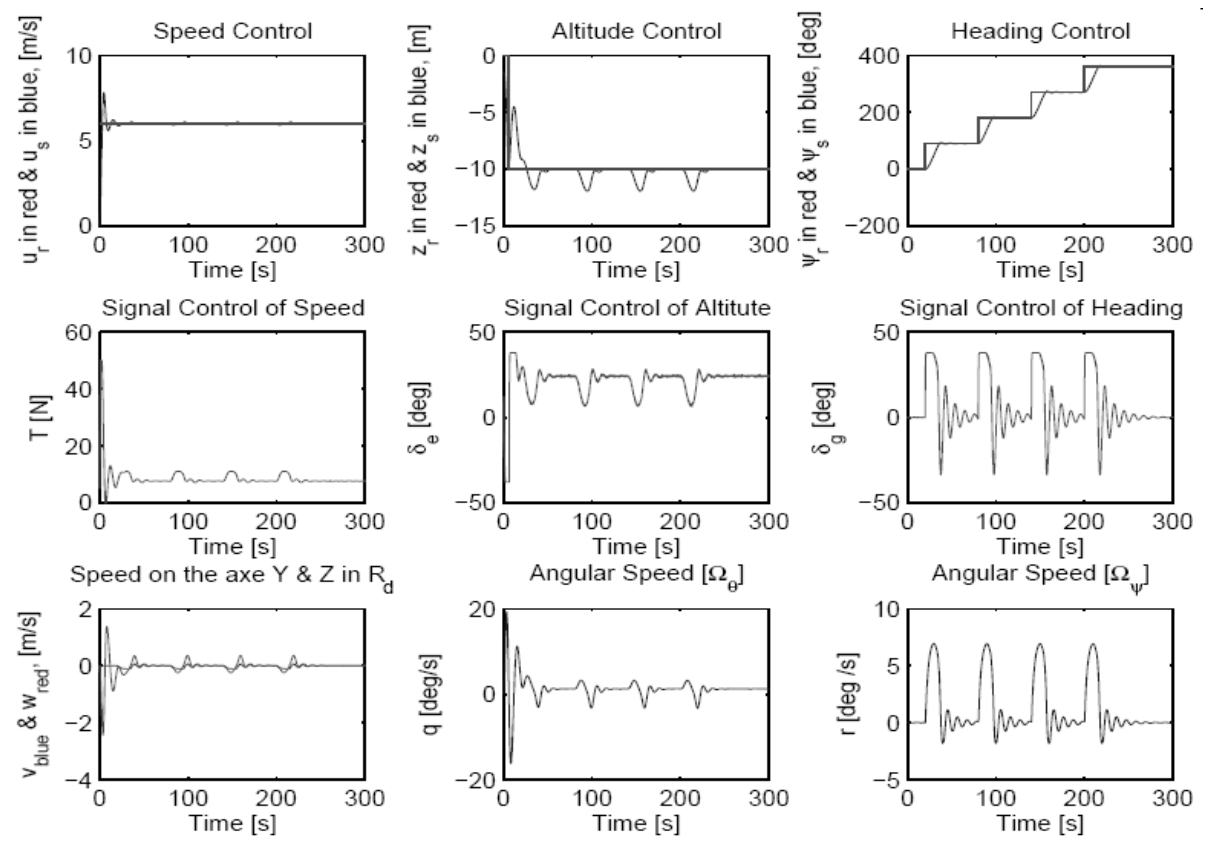

Figura 4. Resultados de los controles GPC's: de arriba a abajo, se presenta la evolución de los sistemas, los controles y las derivadas de las variables de control

Uno de los caminos a seguir por los ingenieros de control durante mucho tiempo ha sido la linealización de las ecuaciones de sistemas no lineales, alrededor de un punto de equilibrio. Relativamente, se han planteado nuevas técnicas según la linealización de los modelos, es decir, según su validez local (en una superficie) o global (todo el espacio de estado).

Cuando un modelo linealizado sobre un punto de operación deja de ser válido, se debe pensar en diferentes modelos linealizados en diferentes puntos de operación. El conjunto de estos modelos linealizados es llamado modelo lineal tangente. Algunos sistemas de control de vuelo de pilotos automáticos para los aviones o sistemas químicos por ejemplo, son realizados con la ayuda de estos modelos.

Para un sistema no lineal la representación de estado puede ser: 


$$
x=f(x, u)
$$

donde $f$ es considerado un campo vectorial parametrizado por la entrada $u$ y sobre una variedad de estado $M$, que puede ser asociada a una superficie (las perturbaciones no se consideran en el planteamiento de la solución). Así, a cada par $(x, u)$ el campo asocia una dirección $f(x, u)$ tangente a la variedad de estado $M$ en el punto $P$ de coordenada $x$. En el caso del dirigible, $f$ es un campo de fuerzas y es modificado por las entradas al sistema que son la propulsión, alerones, vectorización y rotor de cola.

Se puede considerar la aproximación a primer orden del comportamiento de un sistema, como el planteado por la ecuación (31), en la vecindad de un punto de equilibrio $\left(x^{\circ}, u^{\circ}\right)$, utilizando el desarrollo en series de Taylor de $f$.

$$
f(x, u)=f\left(x^{0}, u^{0}\right)+\frac{\partial f}{\partial x}\left(x^{0}, u^{0}\right)\left(x-x^{0}\right)+\frac{\partial f}{\partial u}\left(x^{0}, u^{0}\right)\left(u-u^{0}\right)+\varsigma\left(x-x^{0}, u-u^{0}\right)
$$

despreciando los términos de orden superior e imponiendo $\delta x=x-x^{0}$ y $\delta u=u-u^{0}$, la ecuación (32) puede ser re-escrita como:

$$
\delta \dot{x}=\frac{\partial f}{\partial x}\left(x^{0}, u^{0}\right) \delta x+\frac{\partial f}{\partial u}\left(x^{0}, u^{0}\right) \delta u
$$

que es la representación lineal del sistema sobre el punto de operación. Ahora si se consideran los diferentes modelos en los diferentes puntos de equilibrio, el modelo resultante es el llamado modelo lineal tangente. En Fossard [11] se puede consultar una teoría más profunda y la demostración de la gobernabilidad local. La expresión analítica para el modelo lineal tangente en función del punto de operación se puede definir como:

$$
\delta \dot{x}=F\left(x^{0}, u^{0}\right) \delta x+G\left(x^{0}, u^{0}\right) \delta u
$$

Considerando la ecuación (35) se formula una ley de control $\delta u$ por cualquier método lineal (ubicación de polos, realimentación de estado, control óptimo, etc). Si se formula la ley de control en función del punto de operación y por realimentación de estado con referencia $\delta v$ se tiene:

$$
\delta u=-K\left(x^{0}, u^{0}\right) \delta x+\delta v
$$

Esta ley calculada localmente, debe ser integrada para encontrar la ley de control no lineal y aplicarla a todo el espacio de estado, quedando las ganancias adaptables al punto de operación sin recurrir a estimadores del punto de operación y sin conmutaciones. Esta integración es el paso más difícil y una representación para ella es: 


$$
u=\Omega(x, v)
$$

La noción de los modelos de menor complejidad, presentados anteriormente, permite pensar que el dirigible en la fase de vuelo crucero cumple con un número reducido de limitaciones en el equilibrio, es decir se tiene un equilibrio parcial. Esto facilita la síntesis de leyes de control en diferentes etapas, de tal forma que a cada paso se tenga un número reducido de variables y menor complejidad en los modelos.

Este tipo de reguladores llamados también seudo- compensadores, son desarrollados con la finalidad de fijar los polos del modelo lineal tangente a un valor independiente del punto de operación. Así, se diseñaron los reguladores para los diversos sistemas reducidos. Como requisito antes de realizar algún procedimiento para la obtención de los controles por esta técnica, se debe garantizar que el modelo lineal tangente (caso SISO) dependa sólo de la variable de estado que se va a controlar y que se cumpla la condición de gobernabilidad local $\left(\frac{\partial f}{\partial u}\left(x^{0}, u^{0}\right) \neq 0\right)$. Los desarrollos que se describen a continuación asumen como verdadera esta condición.

\subsubsection{Control de Velocidad}

Para este control se asumió el modelo general de la planta:

$$
m \dot{u}=F_{m} \cos \mu+\frac{1}{2} \rho u^{2} S_{r e f} C_{T t}
$$

Donde $u$ es la variable de estado, $\mu$ el ángulo de vectorización, generalmente próximo a cero en la fase crucero y $F_{m}$ es la señal de control. El punto de equilibrio está dado por:

$$
u_{0}^{2}=\frac{2 F_{m 0} \cos \mu}{\rho S_{r e f} C_{T t}}
$$

y el sistema lineal tangente o de primer orden es:

$$
\begin{gathered}
\delta \dot{u}=\frac{\rho S_{r e f} C_{T t} u_{o}}{m} \delta u+\frac{\cos \mu}{m} \delta F_{m} \\
\delta \dot{u}=a\left(u_{o}\right) \delta u+b\left(u_{o}\right) \delta F_{m}
\end{gathered}
$$

Definiendo la ley de control como:

$$
\delta F_{m}=-K\left(u_{0}, F_{m} 0\right) \delta u+\int\left(\delta u_{r e f}-\delta u\right) d t
$$


tal que el sistema en lazo cerrado, ley de control (42) más planta, satisfagan los requerimientos de desempeño $s^{2}+2 \xi \varpi_{n} s+\varpi_{n}{ }^{2}$ y error en estado estable nulo, se tiene:

$$
\begin{gathered}
k\left(u_{o}\right)=\frac{-2 \xi w_{o} m-\rho S_{r e f} C_{T t} u_{o}}{\cos \mu} m \\
k_{a}\left(u_{o}\right)=\frac{m w_{o}^{2}}{\cos \mu}
\end{gathered}
$$

Realizando la integración de la ley de control (42), se obtiene la siguiente la expresión para el compensador de velocidad:

$$
F_{m}(t)=\frac{-2 \xi w_{o} m u(t)}{\cos \mu}-\frac{1}{2} \frac{\rho S_{r e f} C_{T t} u^{2}(t)}{\cos \mu}+\frac{m w_{o}^{2}}{\cos \mu} \int\left(u_{r e f}(t)-u(t)\right) d t
$$

\subsubsection{Control de Altitud}

Para la obtención de la ley de control de la altitud se efectuaron desarrollos similares al presentado para la velocidad

$$
\begin{aligned}
\delta_{e}= & \frac{w_{n}^{2}}{-u \cos \theta k_{3} \tau_{1} \tau_{2}} \int\left(z_{r e f}-z\right) d t-\frac{\tau_{2}+\tau_{1}+2 \zeta w_{n}}{\tau_{1} \tau_{2} k_{3}} q+ \\
& -\frac{1+2 \zeta w_{n}\left(\tau_{1} \tau_{2}\right)+\tau_{1} \tau_{2}\left(w_{n}^{2}+k_{1}\right)}{\tau_{1} \tau_{2} k_{3}} \theta+\frac{2 \zeta w_{n}+\tau_{1}+\tau_{2}}{\tau_{1} \tau_{2} k_{3} u \cos \theta} z
\end{aligned}
$$

Donde $\zeta, \omega_{n}, \tau_{1}, \tau_{2}$ son parámetros del polinomio en lazo cerrado deseado (desempeño fijado).

Cabe recordar que por disposiciones de los elementos, especialmente la góndola principal, el dirigible no alcanzará un ángulo $\theta$ de 90 grados.

\subsubsection{Control de Dirección}

Para el control de dirección, se puede demostrar que bajo un procedimiento similar a los anteriores, se llega a la ley de control que se presenta a continuación.

$$
\delta_{g}=\frac{w_{n}^{2}}{k_{1} \tau} \int\left(\psi_{r e f}-\psi\right) d t-\frac{2 \xi w_{n} \tau+1}{k_{1} \tau} r-\frac{w_{n}^{2} \tau+2 \xi w_{n}}{k_{1} \tau} \psi
$$




\subsubsection{Resultados de simulación}

La figura 5 presenta los resultados del control FOC, con el mismo modelo e idénticas referencias utilizadas en los controles anteriores.
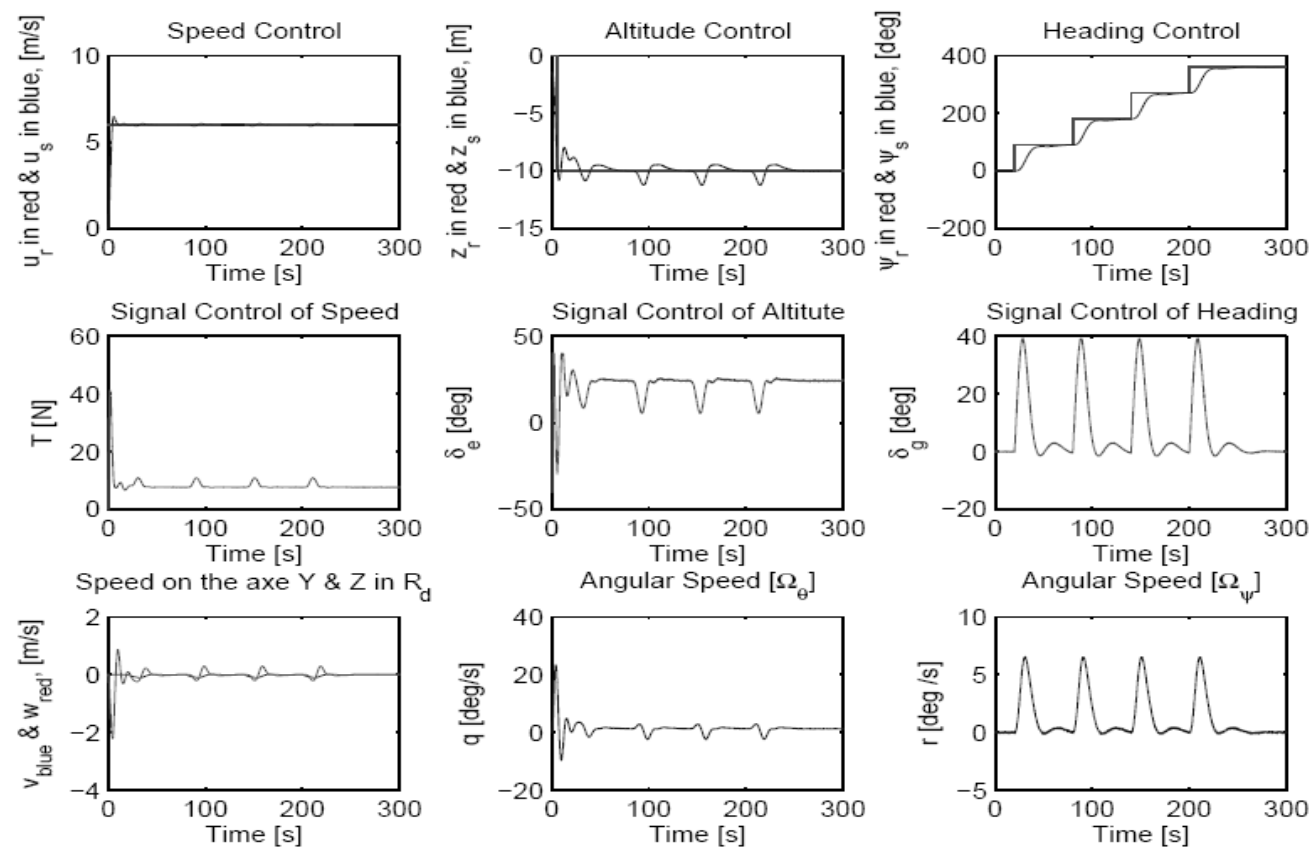

Figura 5. Resultados de los controles NL's: de arriba a abajo, se presenta la evolución de los sistemas, los controles y las derivadas de las variables de control.

\subsection{PRUEBAS CON LOS SISTEMAS REALES}

Las pruebas fueron realizadas sobre las estructures Karma y Uran, pertenecientes respectivamente al laboratorio LAAS/CNRS de Toulouse-Francia y a Uniandes de BogotaColombia.

\subsubsection{Pruebas con Karma y Uran}

La figura 6 muestra el control sobre la velocidad aerodinámica $v_{a}$ y la señal de control enviada a los propulsores $T$, en dicho gráfico se aclara el tipo de control que actúa sobre el sistema: un regulador PID, GPC o NL. Como se aprecia, generalmente el sistema logra seguir la referencia, pero hay momentos cuando la referencia exige demasiado al sistema y se producen saturaciones en la señal de control, por lo cual no logra llegar al punto solicitado. 

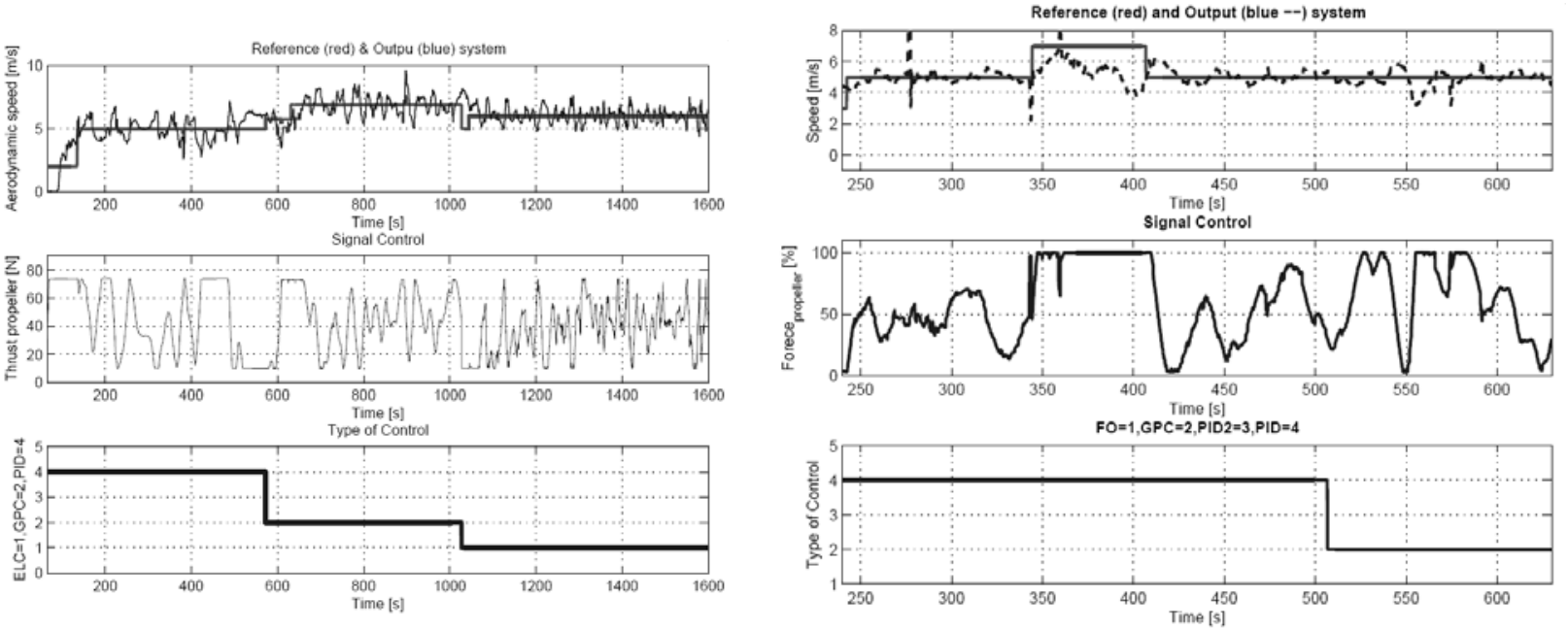

Figura 6. Control de velocidad sobre los sistemas reales: Karma y Uran.

El control de altitud fue probado en Uran, bajo un desplazamiento en condiciones de vuelo crucero. La figura 7 presenta la evolución de la altitud cuando se aplica un regulador GPC. Se observan variaciones fuertes en la señal de control cuando trata de llevar el sistema a la referencia. Estas acciones fuertes pueden ser ocasionadas en parte por la baja precisión del sensor ( $₫ 1)$.

La figura 8 presenta los resultados del control de dirección. Estas pruebas se realizaron sobre la estructura Karma y ponen de manifiesto un aceptable desempeño, logrando llegar a las referencias solicitadas y en un tiempo apropiado a la dinámica de lazo abierto del dirigible.
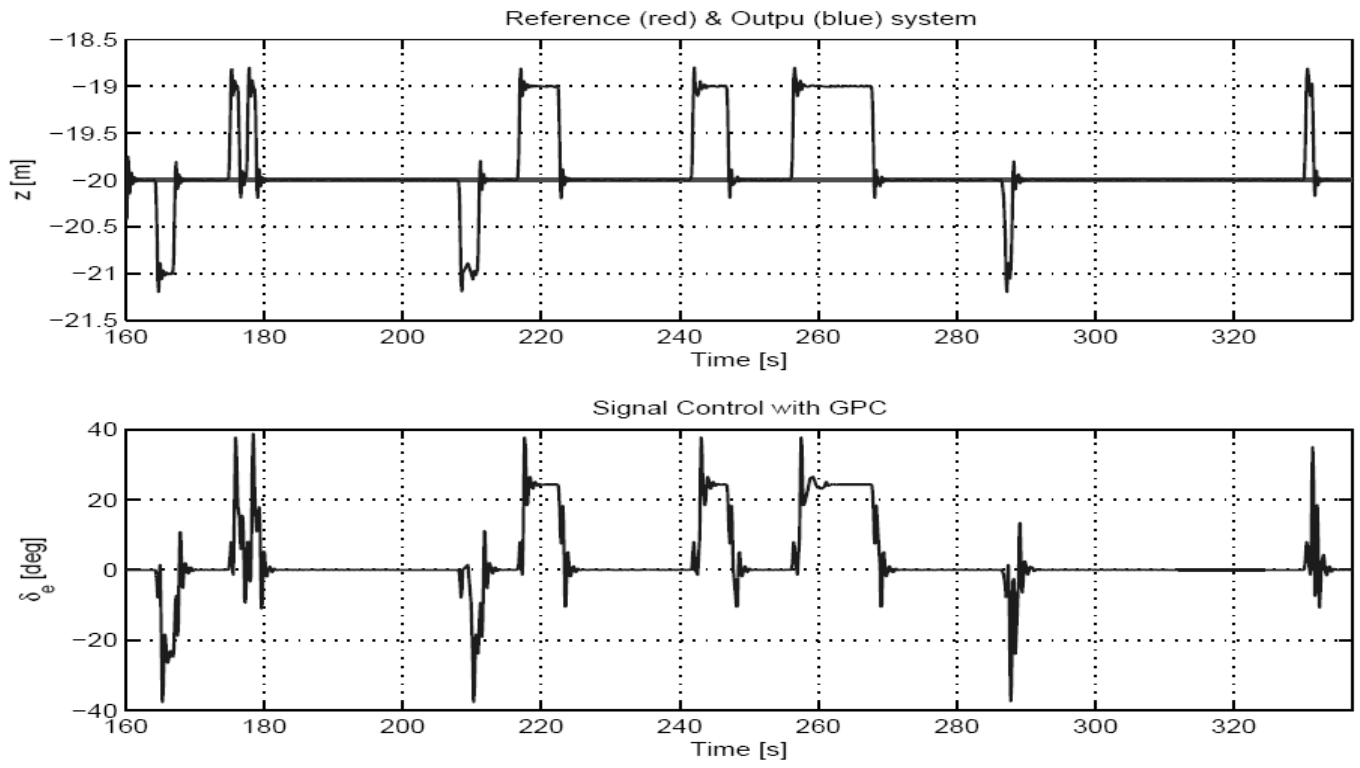

Figura 7. Resultado del control de altitud sobre Uran. 

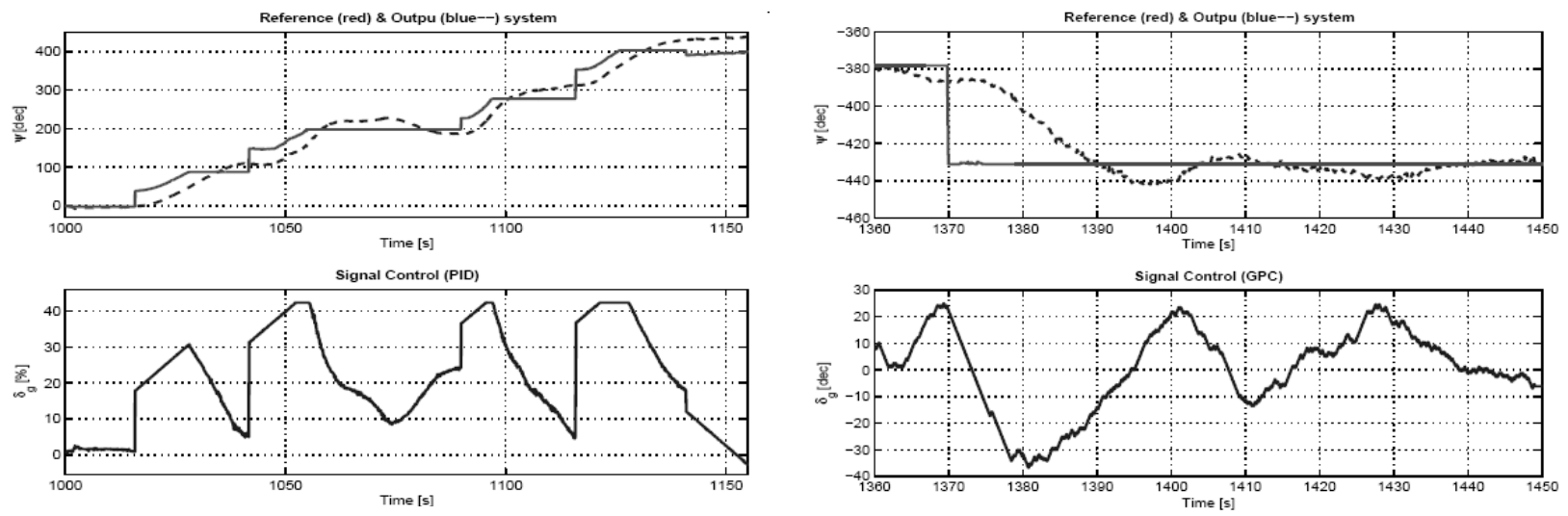

Figura 8. Resultado del control de dirección sobre Karma.

\section{CONCLUSIONES}

Un análisis sobre el comportamiento y el modelo dinámico planteado por la ecuación (3) del dirigible, permitió encontrar un modelo suficientemente representativo de la dinámica del sistema en la fase de vuelo crucero. Bajo las hipótesis mencionadas en la sección 2, el modelo es válido durante el desplazamiento del dirigible en el plano horizontal y con una limitación en los radios de giros. Así, los deslizamientos serán mínimos durante los giros del dirigible y se respetarán las condiciones de vuelo de esta fase.

Se demostró que los modelos reducidos son suficientemente representativos de las principales dinámicas del dirigible y por medio de ellos, se realiza la síntesis de todos los tipos de reguladores aquí presentados.

Un análisis de estabilidad, realizado mediante pruebas en simulación, prueba que los dirigibles se mantendrán siempre en la región de estabilidad, a condición de respetar las dinámicas asociadas a la fase de vuelo crucero.

Las experimentaciones sobre estructuras reales ponen de manifiesto que los reguladores se desempeñan bien en las labores de control de velocidad, altitud y dirección en la fase de vuelo crucero.

Queda abierta la posibilidad de realizar trabajos futuros con reguladores multivariables. Se espera abordar técnicas GPC multivariable, LQ o Backstepping. 


\section{BIBLIOGRAFÍA}

[1] AZINHEIRA, J. R.; CARNEIRO DE PAIVA, E.; RAMOS J.Jr.G. and BUENO, S. S.; Mission path following for an autonomous unmanned airship, IEEE International Conference on Robotics and Automation, San Francisco, April, 2000.

[2] AZINHEIRA, J. R.; Influence of wind speed on airship dynamics, Journal of Guidance, Control, and Dynamics, vol. 24, no. 6, pag. 1116-1124, Nov-Dec, 2002.

[3] BONNET A. and LUNEAU, J.; Identification des coefficients aèrodynamiques du dirigeable AS500 du LAAS, Rapport technique, Etude Hydro-Aèrodynamique, LAAS/ CNRS, March, 2003.

[4] BOSCHMA, J.H.; The development progress of the $\{U\} .\{S\} .\{A\} r m y$ 's SASS LITE, unmanned robot airship, 10th AIAA Lighter-Than-Air Conference and Exhibition, Scottsdale, September, 1993.

[5] CAMACHO, E.F.; BORDONS, C.; Model Predictive Control in the Process Industry Springer, ISBN 3-540-199924-1, 1995.

[6] CLARKE, D.W.; Generalized Predictive Control, Part I, The Basic Algorithm, Automatica, Vol 23, No. 2: pp. 137-148, July, 1987.

[7] DE PAIVA, E.; BUENO, S.; VARELLA GOMES, S.; BERGERMAN, M. and RAMOS, J. A.; Control System Development Environment for AURORA's Semi-Autonomous Robotic Airship, IEEE International Conference on Robotics and Automation, Detroit, Mi. (USA), May, 1999.

[8] DIAZ, H.; SOLAQUE, L. and GAUTHIER, A.; Fuzzy identification and parallel distributed compensation for cruise flight phase of a blimp, $V$ Congreso Internacional Electrónica y Tecnologías de Avanzada - CIETA, Pamplona (Colombia), September, 2006.

[9] ELFES A.; BUENO S.S.; BERGERMAN M.; RAMOS J.G. and VARELLA GOMES, S.B.; Project AURORA: Development of an Autonomous Unmanned Remote Monitoring Robotic Airship, Journal of the Brazilian Computer Society, vol. 4, no. 3, Pag. 70-78, April, 1998.

[10] ELFES, A.; MONTGOMERY, J. F.; HALL, J. L.; JOSHI, S.; PAYNE, J. and BERGH, C. F.; Autonomous flight control for a TITAN exploration AEROBOT, http://wwwrobotics.jpl.nasa.gov/publications/JamesI_Montgomery/2005!_ISAI RASI_Au tonomousFlightControlAerobot.pdf. 
[11] FOSSARD, A.J. and NORMAND-CYROT, D.; Systemes non linl'earies, Copyright Instrument society of America,North Carolina,v. 3, 1993.

[12] HYGPOUNENC, E. and SOUERES, P.; Automatic airship control involving backstepping techniques, LAAS, Report 2159, October, 2002.

[13] HYGOUNENC, E. and SOUERES, P.; A multimode control strategy for autonomous blimp, Report LAAS/CNRS, October, 2002.

[14] 16th LIGHTER-THAN-AIR SYSTEMS TECHNOLOGY Conference and Balloon Systems Conference, Arlington, Va (USA), 2005.

[15] PATIÑO, D.; SOLAQUE L.; LACROIX, S. and GAUTHIER, A.; Estimation of the aerodynamical parameters of an experimental airship, EEE/RSJ IROS 2005 workshop on robot vision for Space Applications, Edmonton, Alberta (Canada), August, 2005.

[16] TUCKERMAN, L. B.; Inertia factors of ellipsoids for use in airship design, N.A.C.A., Report 210, March, 1926.

[17] VARELLA GOMES, S.B. and RAMOS, J.Jr.; Airship dynamic modeling for autonomous operation, Proc. IEEE International Conference on Robotics and Automation, vol. 20, no. 2, Leuven (Belgium), Pag. 3462-3467, May 1998.

[18] ZHENBANG GONG, J.R.; LUO, J. and XIE, S.; A flight control and navigation system of a small size unmanned airship, Proc. IEEE International Conference on Mechatronics and Automation, pag. 1491-1496, Niagara Falls, Canada, July, 2005. 
